\title{
Introduction to the focused issue on music DLs
}

\author{
Nicola Orio • Andreas Rauber • David Rizo
}

Published online: 6 July 2012

(C) Springer-Verlag 2012

This focused issue on Music Digital Libraries of the International Journal of Digital Libraries aims at putting in tighter communication of related research areas that offer plenty of potential for combination and mutual inspiration, yet are only rarely combined from a research perspective: Music Information Retrieval (MIR) and Digital Libraries (DLs) technology.

MIR is a research area that has a primary focus on content-based access and retrieval of music documents. Since the initial approaches based on the query-by-example paradigm, described also as query-by-humming when the musical query was provided by the user directly by singing an excerpt of the melody, MIR research community enlarged its scope to include the recommendation (both of individual music documents and of sequences of songs organized in a playlist), genre classification, artist identification, and automatic tag generation and many other tasks. Other topics related to MIR are more related to music processing, aimed at improved access to music content. To this end, research topics that are related to MIR include segmentation and structure analysis of music documents, both in symbolic and audio formats, track separation and transcription from audio, beat and tempo tracking, instrument identification.

\footnotetext{
N. Orio $(\varangle)$

Department of Cultural Heritage, University of Padua, Padua, Italy

e-mail: orio@dei.unipd.it
}

\author{
A. Rauber \\ Department of Software Technology and Interactive Systems, \\ Vienna University of Technology, Vienna, Austria \\ e-mail: rauber@ifs.tuwien.ac.at \\ D. Rizo \\ Department of Software and Computing Systems, \\ University of Alicante, Alicante, Spain \\ e-mail: drizo@dlsi.ua.es
}

Although DLs technology should play a central role in the development of a MIR system and, on the other hand, MIR results should be of interest for the development of multimedia DLs, the intersection between the two research communities still leaves room for improvement. For example, only about $2 \%$ of the over 1,000 papers presented, since 2000 , at the conferences of the International Society for Music Information Retrieval (ISMIR), mention explicitly DLs in their title. Similar percentages can be found for DLs venues, such as Theory and Practice for Digital Libraries (TPDL, formerly ECDL) and the Joint Conference on Digital Libraries (JCDL, formerly DL). For this reason, we thank the Editorial Board of the International Journal of Digital Libraries for the opportunity to improve the contacts between MIR and DLs through this focused issue.

The contributions presented in this focused issue address a number of important topics in Music Digital Libraries technology. Three papers describe working digital library systems dedicated to different music dimensions. Damm et al. has a focus on improving access to digital audio content through the use of automatic synchronization with other media, such as scanned scores and lyrics. In contrast, Rigaux et al. has as primary application the organization and delivery of symbolic scores, where typical tools for access to digital content are extended by services for score analysis and processing. A novel application of DLs that organize musical ideas for composition is introduced by Bainbridge et al., showing that a combination of MIR techniques within a DL system can be used as a working tool for artistic creation.

The increasing interest towards ethnic music, as an alternative to mainstream music genres, is witnessed by the presence of two papers that address this topic. Strle \& Marolt present an ethno-musicological digital library environment focused on a rich data model exploited by means of MIR tools, showing the interrelation between DLs and MIR above 
discussed, while Canazza focuses on the acquisition stage in any DL, describing the digital restoration of analog audio signals of ethnic music recorded under unfavorable conditions.

Because of their application in the entertainment industry, music DLs can be also a commercial asset and, to this end, Inskip describes how music is searched by professionals through the use of text-based engines, highlighting a number of directions on how to improve music searches. Finally, the paper by Wun-tat Chan et al. presents a novel methodology for MIR, which is included in this focused issue as an example of how MIR approaches can be developed and evaluated; we believe that the fact that the paper deals with widely understandable topics such as rhythm and symbolic notation makes it easily understandable also for readers without a music background and perhaps encourages researchers in DLs to approach this interesting discipline.

We wish to thank all the authors of this focused issue for their valuable contribution, together with the reviewers (belonging to the MIR and to the DLs research communities) for the useful comments that, we believe, make this issue of interest for existing as well as potentially new readers of the International Journal of Digital Libraries, moving closer towards bridging the gap between these communities, supporting transfer of techniques and ideas, and leading to exciting new developments in the area of music digital libraries and beyond. 